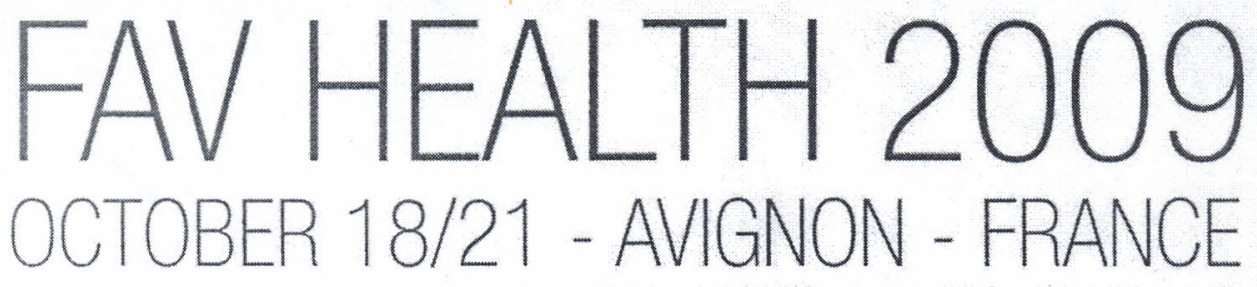

3rd International Symposium on Human Health Effects of Fruits and Vegetables

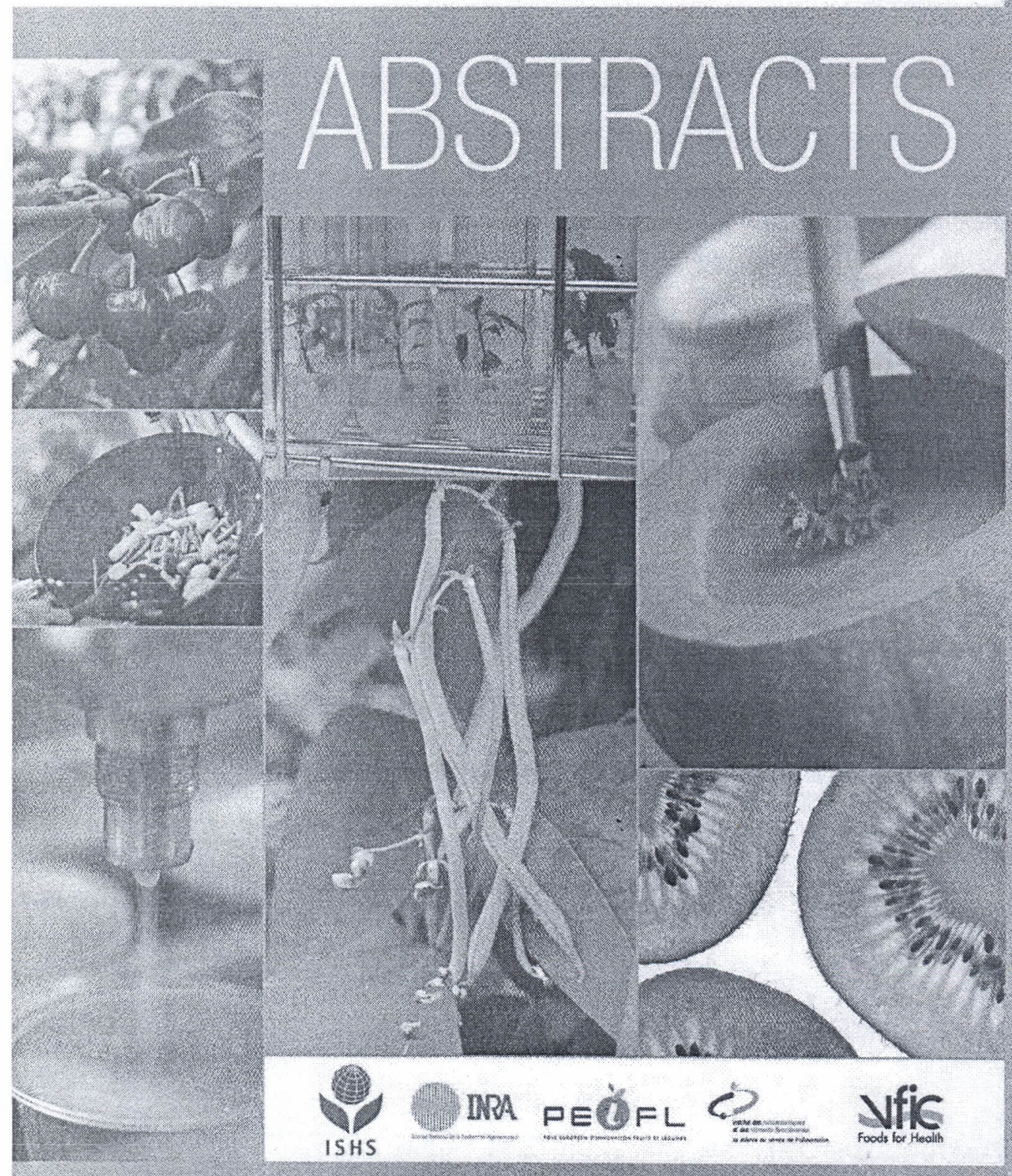




\section{P3-13 Effect of Açai Pasteurization Conditions on its Antioxidant Activity}

Mattietto, R.A.(1); Matta, V.M.(2)

(1)Embrapa Eastern Amazon, Belém, PA, Brazil, rafaella@cpatu.embrapa.br;

(2)Embrapa Food Technology, Rio de Janeiro, RJ, Brazil

The açai tree has been considered as the most important palm of the North region of Brazil, as its fruit pulp consumption has largely increased in the last years being commercialized all over the world mainly due to its composition in bioactive compounds and potentiality as a functional product. This growing market has required the pasteurization of the pulp aiming at the consumers' safety. In this work, it was evaluated the effect of the thermal process conditions on the antioxidant capacity of the açai pulp. The açai fruit, obtained from natural populations of Abaetetuba, $\mathrm{Pa}$, Brazil, was extracted in a mechanical açai depulper, with water addition. The total solids content of the obtained pulp was $13 \%$, which characterizes it as a medium pulp according to the Brazilian legislation. Pulp was homogenized and conducted to the pasteurizer unit. The process was conducted in a continuous small scale system and the tested conditions followed a composed rotational experimental plan, which had temperature $\left(X_{1}\right)$ and time $(X)$ as independent factors and the antioxidant activity as the main response. Results showed that none of the two factors or their interactions was significant at 95\% probability, which means that the antioxidant activity is not significantly affected with time or temperature on proposed levels. Other confidence levels were tested and there were not significant effects and interactions that could permit a model adjustment for this product characteristic, although the tendency plot suggests that the antioxidant activity tends to increase, preferentially, when lower temperatures and higher residence times are applied or with extreme temperatures with very little residence times. The Tukey test (pu0.05) confirmed the experimental plan result showing that there was not a significant difference among most of the tested conditions, being the control sample (without pasteurization) equal to the pasteurized samples except for those obtained at $75^{\circ} \mathrm{C} / 65 \mathrm{~s}, 90^{\circ} \mathrm{C} / 65 \mathrm{~s}$ and $82.5^{\circ} \mathrm{C} / 50 \mathrm{~s}$. It is possible to say that there is a range of time and temperature pasteurization conditions that permits to attain safety and preserve the quality of the açai pulp.

Acknowledgements: To FP6 PAVUC Project Inco-Dev-2-015279, for financial support. 